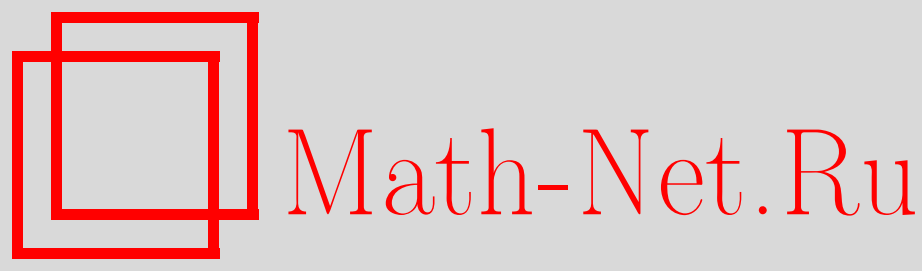

А. В. Карговский, Л. С. Булушова, О.А.Чичигина, Теорема о распределении энергии по степеням свободы для квазистабильного симметричного ангармонического осциллятора, $T M \Phi, 2011$, том 167, номер 2, 273-283

DOI: https://doi.org/10.4213/tmf6639

Использование Общероссийского математического портала Math-Net.Ru подразумевает, что вы прочитали и согласны с пользовательским соглашением http: //www . mathnet.ru/rus/agreement

Параметры загрузки:

IP : 3.85 .73 .92

26 апреля 2023 г., $17: 56: 37$

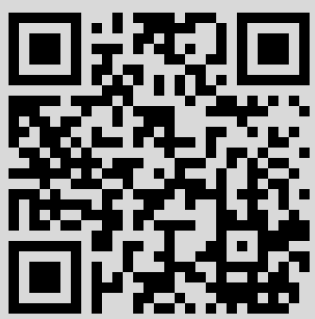


Том 167, № 2

май, 2011

А. В. Карговский*, Л. С. Булушова*, О. А. Чичигина

\title{
ТЕОРЕМА О РАСПРЕДЕЛЕНИИ ЭНЕРГИИ ПО СТЕПЕНЯМ СВОБОДЫ ДЛЯ КВАЗИСТАБИЛЬНОГО СИММЕТРИЧНОГО АНГАРМОНИЧЕСКОГО ОСЦИЛЛЯТОРА
}

\begin{abstract}
Предложен метод описания квазиравновесных процессов в квазистабильном ангармоническом осцилляторе, находящемся под действием теплового шума. Введены эффективные термодинамические параметры, аналогичные соответствующим параметрам равновесной системы, но отличающиеся от них численными значениями. Определен параметр неравновесности системы. Доказана математическая корректность предложенного метода. Получен квазиравновесный аналог теоремы о распределении энергии по степеням свободы.
\end{abstract}

Ключевые слова: квазиравновесное распределение Больцмана, эффективная температура.

\section{1. ВВЕДЕНИЕ}

Квазистабильный симметричный ангармонический осциллятор, находящийся под действием теплового шума, - это система, которая может быть представлена как броуновская частица в потенциальной яме, задающейся степенной зависимостью

$$
U(q)=U_{\min }\left(1-\left(\frac{q}{R}\right)^{\gamma}\right) .
$$

Эта модель представляет особый интерес, так как описывает поведение различных систем в физике, химии и биологии. Такие неравновесные процессы, как диффузия, распад кластера, химические реакции, структурные изменения полимерных молекул, явным образом могут быть описаны с использованием модели броуновской частицы в некотором потенциальном поле [1], [2]. В настоящей работе будем считать, что $\gamma \in\left[e^{-1}, \infty\right)$.

Квазистабильным называется состояние, близкое к равновесному, в котором система может находиться в течение долгого времени: время жизни такого состояния много больше времени корреляций начальных значений. Такое состояние системы

* Московский государственный университет им. М. В. Ломоносова, Москва, Россия. E-mail: kargovsky@yumr.phys.msu.ru 
может определяться некоторым ограничением на определенные параметры, причем достижение этого критического значения в результате тепловых флуктуаций, характеризуемых температурой $T$, должно быть маловероятным событием. Таким параметром может выступать, например, импульс частицы, обладая которым, частица преодолевает потенциальный барьер, и происходит испарение или химическая реакция. Это ограничение приводит к тому, что состояние системы может быть описано не зависящим от времени распределением вероятностей, которое заметным образом отличается от распределения для стабильной системы.

В литературе квазистабильное состояние называется также квазиравновесным, мы будем использовать эти понятия как синонимы. Например, квазиравновесное распределение Больцмана уже применялось ранее для описания диффузии и энтропийных эффектов, аналогичных влиянию так называемого демона Максвелла [3]. Большое время жизни квазиравновесного состояния позволяет нам предположить, что выполнен принцип локального равновесия, и ввести эффективную температуру, характеризующую это состояние. Целью настоящей работы является строгое математическое обоснование корректности метода введения эффективной температуры, доказательство теоремы существования и единственности решения определяющих ее уравнений, дифференцируемости основных термодинамических параметров по этой температуре, рассмотрение асимптотик. Кроме того, нам представляется полезным описать неравновесное состояние в привычных терминах равновесной термодинамики, удобных для использования полученных результатов в приложениях к химии и биологии.

Квазиравновесные состояния в общем случае не моделируются обычными динамическими уравнениями вида $\dot{x}=f(x, t)$, которые удобно исследовать аналитически и/или численно. Условие медленного изменения параметров означает, что $\dot{x} \approx 0$. C точки зрения аналитического исследования это не позволяет отбрасывать малые параметры, а в компьютерном моделировании сильно увеличивает время счета и ошибки.

Рассматриваемая система в течение длительного времени близка к равновесной, но ее нельзя считать строго равновесной, так как мы включаем в рассмотрение неравновесные процессы распада данного состояния. Мы учитываем качественное изменение статистики задачи, описываемое специально введенным параметром неравновесности, в некотором соответствии с подходом из работы [4]. Наш подход позволяет получить результаты в более общем случае, чем стандартные методы решения задачи Крамерса [5], [6].

В настоящей работе прежде всего мы рассмотрим такое квазистабильное состояние системы, в котором ограничение наложено на координаты частицы. Это случай, когда частица достаточно долго находится в потенциальной яме, а затем в результате тепловых флуктуаций совершает выход из нее, пересекая границу ямы $L$, проходящую по гребню потенциального барьера. Полученный результат может быть обобщен на случай, когда ограничение налагается и на импульс.

\section{2. КВАЗИРАВНОВЕСНОЕ РАСПРЕДЕЛЕНИЕ ГИББСА}

2.1. Основные понятия. Пусть для рассматриваемой квазистабильной $(\mathbb{Q} \mathbb{S})$ системы можно ввести фазовое пространство, которое аналогично фазовому пространству гамильтоновой $(\mathbb{S})$ системы; в $\mathbb{S}$-системе отсутствует ограничение, налагаемое границей $L$, в то время как для $\mathbb{Q S}-$ системы конфигурационное простран- 
ство $\mathbf{q}=\left\{x_{1}, x_{2}, \ldots, x_{n}\right\}$ ограничено. Гамильтониан в обоих случаях состоит из суммы кинетической энергии, задаваемой обобщенными импульсами, и потенциальной энергии $U(\mathbf{q})<0$, которая характеризуется глубиной ямы $U_{\min }<0$ и условием $\left.U\right|_{L}=0$. На этом фазовом пространстве для $\mathbb{S}$-системы определена вероятностная мера, а ее равновесная плотность является распределением Гиббса [7]. Аналогично для $\mathbb{Q S}$-системы определяется квазиравновесное распределение Гиббса. При $k T /\left|U_{\min }\right| \rightarrow 0$ все результаты для $\mathbb{Q S}$-системы должны стремиться к соответствующим результатам для $\mathbb{S}$-системы, поскольку в этом случае вероятность выхода частицы из потенциальной ямы стремится к нулю [8].

Квазиравновесное распределение Гиббса мы зададим путем внесения поправок в равновесное распределение Гиббса, пользуясь независимостью распределений по координатам и импульсу. Будем характеризовать изменения, вносимые ограничением по координатам, некоторым параметром $\alpha$, а ограничения по импульсу - параметром $\lambda$. Квазиравновесное распределение Гиббса для такого состояния получается обобщением введенного ранее в работах [8]-[10] квазиравновесного распределения Больцмана. Его также можно считать условным распределением вероятностей при условии, что выход из потенциальной ямы еще не произошел:

$$
w(\mathbf{q}, \mathbf{p})=\frac{C}{\Omega} \exp \left(\frac{\bar{U}-\alpha U(\mathbf{q})-\lambda \mathbf{p}^{2} / 2 m}{k T}\right),
$$

где $\Omega$ - объем ямы, $k$ - постоянная Больцмана, $C$ - константа нормировки распределения по импульсам. Здесь усреднение подразумевается по распределению $w(\mathbf{q}, \mathbf{p})$. Отличие параметров $\alpha$ и $\lambda$ от единицы свидетельствует о неравновесности системы.

2.2. Квазиравновесное распределение Больцмана. Мы сосредоточимся на случае $\lambda=1$, т. е. на случае, когда ограничение по импульсам отсутствует, и будем использовать квазиравновесное распределение Больцмана, задаваемое двумя параметрами $\alpha$ и $\bar{U}$.

Случай $\alpha=1$ соответствует равновесному распределению Гиббса. Аналогично последнему распределение (1) распадается на распределение Максвелла для импульсов и квазиравновесное распределение Больцмана для координат. Устойчивое и неустойчивое распределения отличаются только перенормировкой температуры. Назовем величину $T_{\text {in }}=T / \alpha$ внутренней эффективной температурой. Величины $\alpha$ и $\bar{U}$ определяются из системы уравнений

$$
\begin{aligned}
\int e^{-\alpha U(\mathbf{q}) / k T} d \mathbf{q} & =\Omega e^{-\bar{U} / k T}, \\
\frac{\int U(\mathbf{q}) e^{-\alpha U(\mathbf{q}) / k T} d \mathbf{q}}{\int e^{-\alpha U(\mathbf{q}) / k T} d \mathbf{q}} & =\bar{U}
\end{aligned}
$$

Первое из этих уравнений получается из условия нормировки, а второе - из того факта, что $\bar{U}$ является математическим ожиданием величины $U$, где усреднение произведено по координатам.

Введем следующие безразмерные переменные: $\beta=-U_{\min } / k T$ - обратная темпеpaтура, $\zeta=-U_{\min } / k T_{\text {in }}$ - обратная внутренняя температура, $\mathbf{r}=\mathbf{q} / R-$ координата, $\omega=\Omega / R^{d}$ - объем ямы, где $d$ - размерность пространства, $\bar{u}=-\bar{U} / U_{\min }-$ средняя 
потенциальная энергия, $u(\mathbf{r})=-U(\mathbf{r}) / U_{\min }$. Они удовлетворяют неравенствам

$$
\begin{aligned}
& \beta \geqslant 0, \quad \zeta=\beta \alpha \geqslant 0, \quad 0 \leqslant r \leqslant 1, \\
& \omega>1, \quad-1<\bar{u}<0, \quad-1 \leqslant u(\mathbf{r}) \leqslant 0 .
\end{aligned}
$$

Тогда система (2) перепишется в виде

$$
\begin{aligned}
\int e^{-\zeta u(\mathbf{r})} d \mathbf{r} & =\omega e^{-\beta \bar{u}}, \\
\int u(\mathbf{r}) e^{-\zeta u(\mathbf{r})} d \mathbf{r} & =\omega \bar{u} e^{-\beta \bar{u}},
\end{aligned}
$$

где потенциальная энергия может быть представлена как

$$
u(r)=-1+r^{\gamma} .
$$

В случае двумерной симметричной ямы $\omega=\pi$, и интегрирование по объему можно свести к интегрированию по радиусам.

2.3. Квазиравновесный конфигурационный интеграл. Введем на интервале $[0, \infty)$ функцию

$$
Q(\zeta)=2 \int_{0}^{1} e^{-\zeta u(r)} r d r .
$$

Она представляет собой координатную часть статистической суммы, или конфигурационный интеграл. Здесь учтено то, что мы рассматриваем двумерную симметричную потенциальную яму. В дальнейшем мы не будем писать пределы интегрирования.

ЛЕмма 1. Производная $d Q / d \zeta$ существует и положительна при любых $\zeta \geqslant 0 u$ $-1 \leqslant u(r) \leqslant 0$.

ДокАЗАТЕЛЬСТво. Исследуем функцию $Q(\zeta)$ на монотонность на заданном интервале. Функция дифференцируема, так как функция $e^{-\zeta u(r)}$ дифференцируема. Дифференцируя, получаем

$$
\frac{d Q}{d \zeta}=-2 \int u(r) e^{-\zeta u(r)} r d r>0 .
$$

Предельное значение $Q(\zeta)$ определено для любых $u:$ мы имеем $\lim _{\zeta \rightarrow \infty} Q(\zeta)=+\infty$. Таким образом, функция $Q(\zeta)$ монотонно возрастает на всей положительной полуоси и принимает все значения в диапазоне $[1, \infty)$. Лемма доказана.

Указанный случай мы будем рассматривать в дальнейшем, а потом обобщим его на системы с произвольной размерностью.

Для потенциала (4) можно получить явное выражение для конфигурационного интеграла:

$$
Q(\zeta)={ }_{1} F_{1}\left(1,1+\frac{2}{\gamma} ; \zeta\right)
$$

где ${ }_{1} F_{1}(a, b ; z)$ - вырожденная гипергеометрическая функция. 
2.4. Средняя потенциальная энергия. Определим среднюю потенциальную энергию, отнесенную к $U_{\min }$, как функцию переменной $\zeta$, заданную на интервале $[0, \infty)$ равенством

$$
\bar{u}(\zeta)=\frac{2}{Q(\zeta)} \int u(r) e^{-\zeta u(r)} r d r .
$$

ЛЕмма 2. Производная $d \bar{u} / d \zeta$ существует и неположительна при любъх $\zeta \geqslant 0$ $u n p u-1 \leqslant u(r) \leqslant 0$.

ДокАзАтЕЛЬство. Числитель и знаменатель в правой части (6) являются дифференцируемыми функциями переменной $\zeta$, причем $Q(\zeta) \neq 0$. Производя дифференцирование, получаем

$$
\frac{d \bar{u}}{d \zeta}=-\overline{u^{2}}+\bar{u}^{2} \leqslant 0
$$

Здесь учтено, что $\overline{u^{2}} \geqslant \bar{u}^{2}$. Предельные значения функции $\bar{u}(\zeta)$ также определены для любых $u$ :

$$
\bar{u}(0)=\frac{2}{Q(0)} \int u(r) r d r<0, \quad \lim _{\zeta \rightarrow \infty} \bar{u}(\zeta)=-1 .
$$

Таким образом, функция $\bar{u}(\zeta)$ монотонно убывает на всей положительной оси и принимает все значения в диапазоне $(-1, \bar{u}(0)]$. Лемма доказана.

Выполняются также известные в равновесной статистической физике соотношения, например

$$
\frac{d Q}{d \zeta}=-Q \bar{u} .
$$

Можно провести интегрирование в (6) и получить

$$
\bar{u}(\zeta)=-\frac{2}{\gamma \zeta Q}+\frac{2}{\gamma \zeta}-1 .
$$

Из этого представления видно, что средняя потенциальная энергия, отнесенная к $U_{\min }$, стремится к -1 при малых температурах, как $(2 / \gamma \zeta)-1$. Очевидно, что при низких температурах средняя энергия равна минимальной.

\section{5. Теорема существования и единственности решения.}

Теорема. Решение первого уравнения системы (3) существует и единственно при $u(r)=-1+r^{\gamma} u \beta \geqslant 0$.

ДокАзАТЕЛЬство. Введем функцию $\beta(\zeta)$, выразив $\beta$ из первого уравнения в (3) и использовав (5):

$$
\beta(\zeta)=-\frac{1}{\bar{u}(\zeta)} \ln Q(\zeta)
$$

Здесь мы учли, что $\bar{u} \neq 0$. Докажем, что производная $d \beta / d \zeta$ существует, и при любых $\zeta$ и $u$ справедливо неравенство

$$
\frac{d \beta}{d \zeta} \leqslant 1
$$


Для доказательства существования производной $d \beta / d \zeta$ учтем, что $\bar{u}(\zeta)$ и $Q(\zeta)$ являются дифференцируемыми функциями, не принимающими нулевого значения. Следовательно, функция $\beta(\zeta)$ дифференцируема. Ее предельные значения выражаются через предельные значения этих функций:

$$
\beta(0)=0, \quad \lim _{\zeta \rightarrow \infty} \beta(\zeta)=\infty
$$

Производя дифференцирование уравнения (10) по $\zeta$ и учитывая равенство (8), получаем

$$
\frac{d \beta}{d \zeta}=1-\frac{\beta}{\bar{u}} \frac{d \bar{u}}{d \zeta} \leqslant 1
$$

Таким образом,

$$
\beta(\zeta) \leqslant \zeta
$$

Продифференцировав равенство (9), получим выражения для первой и второй (потребуется в дальнейшем) производной функции $\bar{u}$ :

$$
\begin{aligned}
\frac{d \bar{u}}{d \zeta} & =-\frac{1+\bar{u}}{\zeta}-\frac{2 \bar{u}}{\gamma \zeta Q}, \\
\frac{d^{2} \bar{u}}{d \zeta^{2}} & =-\left(\frac{2}{\gamma \zeta Q}+\frac{2}{\zeta}\right) \frac{d \bar{u}}{d \zeta}-\frac{2 \bar{u}^{2}}{\gamma \zeta Q} .
\end{aligned}
$$

Предельные значения производной функции $\beta(\zeta)$ определяются следующими выражениями:

$$
\left.\frac{d \beta}{d \zeta}\right|_{\zeta=0}=1, \quad \lim _{\zeta \rightarrow \infty} \frac{d \beta}{d \zeta}=1 .
$$

Для больших температур этот результат следует из формул (12) и (7) для предельных значений $\beta(0)$ и $\bar{u}(0)$. При малых температурах формула (15) позволяет оценить выражение $d \bar{u} / d \zeta$ во втором слагаемом в соотношении (13):

$$
\lim _{\zeta \rightarrow \infty} \frac{d \bar{u}}{d \zeta}=-\frac{2}{\gamma \zeta^{2}}
$$

Оценим второе слагаемое в (13), учитывая неравенство (14):

$$
0<\frac{\beta}{\bar{u}} \frac{d \bar{u}}{d \zeta}=\frac{\beta}{\bar{u}} \frac{2}{\gamma \zeta^{2}} \leqslant \frac{\zeta}{\bar{u}} \frac{2}{\gamma \zeta^{2}} \rightarrow+0 .
$$

Докажем, что функция $\beta(\zeta)$ монотонно возрастает, т. е. $d \beta / d \zeta>0$ при любых $\zeta$ и $\gamma \geqslant e^{-1}$. Предположим, что $\beta(\zeta)$ имеет экстремумы, тогда в соответствии с ее предельными значениями (12) и предельными значениями ее производной (17) в силу непрерывности она должна иметь четное количество экстремумов (как минимум два). Найдем вторую производную $\beta^{\prime \prime}(\zeta)$ в точке экстремума и докажем, что она всегда больше нуля:

$$
\frac{d^{2} \beta}{d \zeta^{2}}=-\frac{\beta}{\bar{u}}\left[\frac{d^{2} \bar{u}}{d \zeta^{2}}-\frac{1}{\bar{u}}\left(\frac{d \bar{u}}{d \zeta}\right)^{2}\right]>0 .
$$




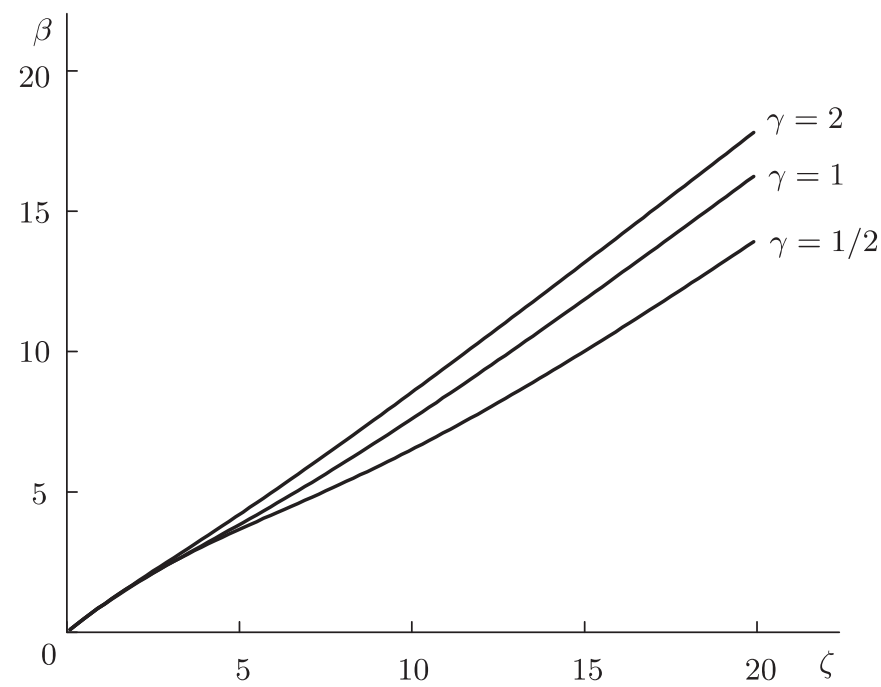

Рис. 1. Зависимость $\beta(\zeta)$ при различных значениях $\gamma$.

Подставляя в левую часть этого неравенства производные энергии из равенств (15) и (16), получаем соотношение

$$
-\frac{2 \ln Q}{\gamma Q} \geqslant-\frac{2 e^{-1}}{\gamma} \geqslant-2>\frac{1}{\bar{u}}-1,
$$

доказывающее, что неравенство действительно верно. Итак, если экстремумы функции существуют, то они все являются минимумами, а это противоречит граничным значениям самой функции и ее производных, следовательно, верно обратное. На положительной оси функция $\beta(\zeta)$ принимает все значения в диапазоне $[0, \infty)$.

Из монотонности и дифференцируемости функции $\beta(\zeta)$ следует существование монотонной функции $\zeta(\beta)$, т. е. каждому значению $\beta$ соответствует одно значение $\zeta$. Теорема доказана.

На рис. 1 показана функция $\beta(\zeta)$ при различных значениях $\gamma$.

2.6. Параметр неравновесности. Рассмотрим отношение $\alpha=\zeta / \beta$ реальной температуры и внутренней эффективной температуры. Сформулируем два следствия из теоремы существования и единственности.

СледСтвиЕ 1. При указанных свойствах потенциальной ямы $\alpha \geqslant 1$ для любъх $\beta$.

ДокАзАтельство. Согласно неравенству (14) $\beta \leqslant \alpha \beta$, поэтому функция $\alpha \beta$ возрастает быстрее чем $\beta$. При $\beta=0$ они совпадают в силу (17). Следовательно, $\alpha \geqslant 1$.

СЛЕДСТВИЕ 2. При приближении состояния системы $\kappa$ равновесному $(\zeta \rightarrow \infty)$ разница между равновесным и квазиравновесным распределениями исчезает:

$$
\lim _{\beta \rightarrow \infty} \alpha=1 \text {. }
$$




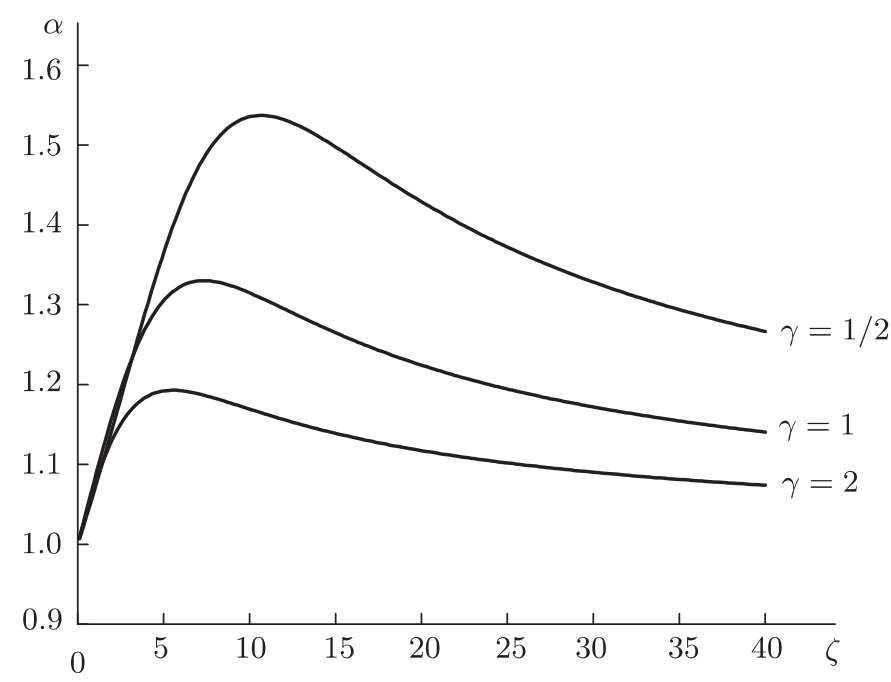

Рис. 2. Зависимость параметра неравновесности $\alpha(\zeta)$ при различных значениях $\gamma$.

ДоказАтельство. Второе предельное значение в (17) говорит о том, что при $\zeta \rightarrow \infty$ неравенство (11) превращается в равенство, которое можно проинтегрировать по $\zeta$ и получить $\beta \alpha=\beta+$ const, что дает $\alpha \rightarrow 1$.

Несложно получить и аналитическое выражение для параметра неравновесности:

$$
\alpha=\frac{\zeta}{1+2 / \gamma} \cdot \frac{{ }_{1} F_{1}(2,2+2 / \gamma ; \zeta)}{{ }_{1} F_{1}(1,1+2 / \gamma ; \zeta) \ln _{1} F_{1}(1,1+2 / \gamma ; \zeta)} .
$$

На рис. 2 изображена зависимость параметра неравновесности от $\zeta$ при различных значениях $\gamma$.

Мы показали, что внутренняя эффективная температура всегда меньше реальной, и их разность имеет максимум. Неравенство $T_{\text {in }}<T$ можно интерпретировать следующим образом. Тепловые флуктуации термостата, действующие на частицу в яме и характеризуемые температурой $T$, можно условно разделить на слабые, не приводящие к выходу из ямы, и сильные, выбивающие частицу из ямы. Иными словами, сильные флуктуации приводят к разрушению данного квазиравновесного состояния, а слабые - нет. Внутренняя температура связана только со слабыми флуктуациями. Одна из возможных интерпретаций данного результата заключается в том, что эффективная глубина ямы в $\alpha$ раз больше чем $U_{\min }$. Подробнее этот эффект описан в работе [11].

\section{3. КВАЗИРАВНОВЕСНЫЙ АНАЛОГ ТЕОРЕМЫ О РАСПРЕДЕЛЕНИИ ЭНЕРГИИ ПО СТЕПЕНЯМ СВОБОДЫ ДЛЯ АНГАРМОНИЧЕСКОГО ОСЦИЛЛЯТОРА}

$\mathrm{B} \mathbb{S}$-системе распределение энергии по степеням свободы вытекает из известного выражения

$$
\overline{z_{i} \frac{\partial H(\mathbf{z})}{\partial z_{j}}}=k T \delta_{i j}
$$


где $\delta_{i j}$ - символ Кронекера, $\mathbf{z}=(\mathbf{q}, \mathbf{p})$ [12], [13]. Данное соотношение непосредственно следует из распределения Гиббса и того факта, что это распределение стремится к нулю при граничных (или бесконечно больших) значениях канонических переменных. Для стабильного ангармонического осциллятора степени $\gamma$ и размерности $d$ получается хорошо известное соотношение

$$
\bar{U}=U_{\min }+\frac{d k T}{\gamma} .
$$

В нашем случае $\mathbb{Q S}$-системы нельзя считать, что функция распределения вероятностей обращается в ноль на границе фазового пространства. Это приводит к появлению дополнительного слагаемого наряду с заменой температуры на эффективную температуру. Результат также зависит от того, как именно наложено ограничение на координаты. Мы рассмотрим случай, когда ограничение налагается на модуль радиус-вектора, $q \leqslant R$, и случай, когда модули проекций радиус-вектора, т. е. обобщенных координат, ограничены некоторым значением, $x_{i} \leqslant M$.

3.1. Сферически-симметричная потенциальная яма. Легко проверить, что все результаты, полученные нами для двумерной симметричной ямы, обобщаются на случай любой размерности $d$ пространства. Для этого нужно все выражения вида $2 / \gamma$ в приведенных выше формулах заменить на $d / \gamma$. Теорема существования и единственности решения будет также справедлива, если потребовать $\gamma \geqslant d e^{-1} / 2$.

Запишем уравнение для внутренней энергии (9) в размерных единицах в наиболее удобной для практических применений форме:

$$
\bar{U}=U_{\min }+\frac{d k T}{\gamma \alpha}-\frac{d k T}{\gamma \alpha} e^{\bar{U} / k T} .
$$

Здесь последнее слагаемое в правой части является малым параметром. Поэтому можно в нулевом приближении вычислить среднюю энергию, пренебрегая этим слагаемым, а потом уточнить, применяя метод последовательных приближений. Экспонента в правой части (19) определяет вероятность выхода из потенциальной ямы и является малой по условию квазиравновесности.

Выражение (19) допускает ясную физическую интерпретацию в терминах среднего времени выхода частицы из потенциальной ямы. Среднее время выхода (так называемое время Крамерса [5]) вычислено в работах [3], [8] и может быть выражено через вероятность выхода в единицу времени:

$$
\frac{1}{\tau}=\bar{v}^{+} L w(L)
$$

где $\bar{v}^{+}$- средняя положительная проекция скорости частицы на направление внешней нормали, $w(L)$ - квазиравновесное распределение Больцмана (1) на границе ямы. Подставляя эти выражения в (20), получаем

$$
\tau=\frac{t_{\text {out }}}{2} e^{-\bar{U} / k T}
$$

где введено время $t_{\text {out }}=R / \bar{v}^{+}$, характеризующее прохождение барьера. Здесь мы используем возможность описания процессов в квазистабильном состоянии с помощью тех же формул, что и для стабильного состояния с учетом эффективной температуры. 
Для частного случая одномерной параболической ямы выражение (20) совпадает с хорошо известным законом Аррениуса с единичным префактором [6].

С учетом равенства (21) выражение (19) может быть записано в виде

$$
\bar{U}=U_{\min }+\frac{d k T_{\text {in }}}{\gamma}\left(1-\frac{t_{\text {out }}}{2 \tau}\right) .
$$

Интересно сравнить это выражение для средней энергии со значением, которое можно получить для равновесной системы согласно теореме о распределении энергии по степеням свободы. Во-первых, в (22), очевидно, используется внутренняя температура $T_{\text {in }}$. Во-вторых, часть потенциальной энергии внутри ямы тратится на движение через потенциальный барьер, эти потери энергии характеризуются отношением времени перехода и времени пребывания в яме. Приведенное соотношение не противоречит второму закону термодинамики, так как свободная энергия обеих систем одинакова, а разница энтропий объясняется внесением в квазиравновесную систему дополнительной информации, этот вопрос подробно рассматривается в работе [3] и монографии [14].

3.2. Случай независимых координат. В этом случае потенциальная энергия разбивается на сумму независимых слагаемых вида

$$
U\left(x_{i}\right)=U_{\min }\left(1-\left(\frac{x_{i}}{M}\right)^{\gamma}\right) .
$$

Мы можем непосредственно использовать соотношение (18), где интегрирование по частям приводит к формуле, аналогичной (19):

$$
\overline{U_{i}}=U_{\min }+\frac{k T}{\gamma \alpha}-\frac{k T}{\gamma \alpha} e^{\overline{U_{i}} / k T}=U_{\min }+\frac{k T_{\text {in }}}{\gamma}\left(1-\frac{t_{\text {out }}}{2 \tau}\right) .
$$

\section{4. ЗАКЛЮЧИТЕЛЬНЫЕ ЗАМЕЧАНИЯ}

Полученный результат для обобщенных координат в случае гармонического осциллятора $(\gamma=2)$ можно применить к обобщенным импульсам, так как они входят в гамильтониан квадратичным образом. Ограничение может быть наложено как на модуль импульса, так и на его проекции. В любом случае мы получаем равенство средней кинетической энергии и внутренней температуры $T / \lambda$.

Получить условное распределения вероятностей для ангармонического осциллятора при условии, что его координаты не превосходят некоторого граничного значения, в общем случае весьма сложно. Однако в предположении малой вероятности достижения этой границы удается записать такое распределение, пользуясь аналогией с распределением Больцмана. Параметры этого нового распределения, эффективная температура и средняя потенциальная энергия, являются однозначно определенными и непрерывными при всех значениях температуры. Теорема о распределении энергии квазиравновесной системы по степеням свободы позволяет представить среднюю потенциальную энергию в виде, аналогичном равновесному случаю, т. е. в виде линейного по эффективной температуре слагаемого с таким же коэффициентом и некоторой малой добавки, зависящей от отношения времени перехода ко времени ожидания. 


\section{Список литературы}

[1] A. Einstein, Investigations of the Theory of the Brownian Movement, Dover, New York, 1956.

[2] Р. Л. Стратонович, Случайные прочессы в динамических системах, РХД, М., Ижевск, 2009.

[3] О.А. Чичигина, ЖЭТФ, 116:1 (1999), 57-66.

[4] P. S. Landa, Yu. I. Neimark, P. V. E. McClintock, J. Stat. Phys., 125:3 (2006), 593-620.

[5] H. A. Kramers, Physica, 7:4 (1940), 284-304.

[6] V. I. Mel'nikov, Phys. Rep., 209:1-2 (1991), 1-71.

[7] В. В. Козлов, Д. В. Трещев, ТМФ, 134:3 (2003), 388-400.

[8] О.А. Чичигина, "Квазиравновесное решение задачи Крамерса", Памяти А. Н. Малахова: сборник научных трудов, ред. А. И. Саичев, Талам, Н. Новгород, 2000, 98-108.

[9] О. А. Чичигина, А. В. Нетребко, Изв. вузов. Прикл. нелин. динам., 9:3 (2001), 85-94.

[10] Л. С. Булушова, О.А. Чичигина, Вестн. Моск. ун-та. Сер. физ., астр., 2010, №2, $31-36$.

[11] Я. Г. Синай, ТМФ, 121:1 (1999), 110-116.

[12] В. В. Козлов, Нелин. динам., 3:2 (2007), 123-140.

[13] Р. Л. Стратонович, М. С. Полякова, Элементы молекулярной физики, термодинамики и статистической физики, МГУ, М., 1981.

[14] Р. Л. Стратонович, Теория информации, Советское радио, М., 1975.

Поступила в редакцию 3.06.2010, после доработки 19.11.2010 